\title{
Preparação e caracterização do carvão ativado produzido a partir do endocarpo de Tucumã (Astrocaryum aculeatum)
}

O processo de adsorção é bastante importante na remoção de poluentes em meios fluidos, principalmente em efluentes líquidos, visto que o sistema de tratamento não é suficiente para certos compostos. O carvão ativado é um poderoso material adsorvente de baixo custo e com possibilidade de ser produzido a partir de resíduos agroindustriais, como o endocarpo de tucumã, fruto bastante presente na região amazônica do Brasil. A matéria-prima ativada com ZnCl2 e carbonizada a $500{ }^{\circ} \mathrm{C}$ resultou num carvão ativado com 6,12 e 7,70 \% de umidade e cinzas, respectivamente. O CATC apresentou grupos de superfície predominantemente ácidos e pH no ponto de carga zero igual 5,54, indicando grande potencial na aplicação de diversos poluentes aquáticos.

Palavras-chave: Tucumã; Caracterização; Adsorção.

\section{Preparation and characterizations of activated carbon from Tucumã endocarp (Astrocaryum aculeatum)}

The adsorption process is very important in removing pollutants in fluid media, especially in liquid effluents, since the treatment system is not sufficient for certain compounds. Activated carbon is a powerful low-cost adsorbent material with the possibility of being produced from agro-industrial residues, such as the tucumã endocarp, a fruit widely present in the Amazon region of Brazil. The raw material activated with $\mathrm{ZnCl} 2$ and carbonized at $500{ }^{\circ} \mathrm{C}$ resulted in an activated carbon with 6.12 and $7.70 \%$ moisture and ash, respectively. CATC has predominantly acidic surface groups and pH at point zero charge equal to 5.54 , indicating great potential in the application of various aquatic pollutants.

Keywords: Tucumã; Characterization; Adsorption.

Topic: Engenharia Química

Reviewed anonymously in the process of blind peer
Received: 05/06/2021

Approved: 26/06/2021
Walber José Pereira Costa (iD

Universidade Federal do Pará, Brasil

http://lattes.cnpq.br/4664698269650403

http://orcid.org/0000-0002-9235-7896

walberi.pereira@gmail.com

Lênio José Guerreiro de Faria

Universidade Federal do Pará, Brasil

http://lattes.cnpq.br/7428609361678173

http://orcid.org/0000-0002-9534-9998

lenio@ufpa.br

Erika Milene Pinto de Sousa

Universidade Federal Rural da Amazônia, Brasil

http://lattes.cnpq.br/6891150911435999

http://orcid.org/0000-0002-8543-6385

erika.sousa@ufra.edu.br
Referencing this:

COSTA, W. J. P.; FARIA, L. J. G.; SOUSA, E. M. P.. Preparação e caracterização do carvão ativado produzido a partir do endocarpo de Tucumã (Astrocaryum aculeatum). Revista Ibero Americana de Ciências Ambientais, v.12, n.6, p.405-410, 2021. DOI: http://doi.org/10.6008/CBPC2179-6858.2021.006.0034 


\section{INTRODUÇÃO}

Entende-se como adsorção o processo onde há o aumento da concentração de um determinado componente sobre a superfície de um material sólido e deseja-se que este possua elevada área superficial para facilitar a adsorção. Mecanismos físicos e/ou químicos são responsáveis por esse processo de separação, sendo eles: fisissorção e quimissorção). Um dos materiais adsorventes mais utilizados é o carvão ativado.

O carvão é um material biológico obtido a partir da queima/combustão da matéria-prima, obtendose um produto carbonáceo predominantemente amorfo. Essa combustão pode se dar de forma completa, parcial ou através da decomposição térmica de várias substâncias carbonáceas, assim, produzindo um material de estrutura porosa e elevada área superficial.

Atualmente, enxerga-se a produção de carvão ativado a partir de resíduos agroindustriais visando o reaproveitamento da matéria-prima e aplicando na remoção de poluentes do meio ambiente como os contaminantes emergentes, principalmente os farmacêuticos.

\section{MATERIAS E MÉTODOS}

\section{Produção do carvão ativado}

Os caroços (endocarpo) do fruto de tucumã foram recebidos como resíduo por parte de alunos de graduação em Engenharia Química que visava trabalhar apenas com a amêndoa do mesmo (parte mais interna do fruto). O material já estava seco e foi submetido trituração em moinho de facas para a obtenção de frações menores.

$\mathrm{O}$ caroço já seco e triturado foi submetido à imersão em solução de $\mathrm{NaOH}(0,5 \mathrm{~N})$ para remover possíveis contaminantes orgânicos e resíduos que influenciassem nos processos seguintes. Após $24 \mathrm{~h}$ o material foi retirado da solução de $\mathrm{NaOH}$ e lavado com água destilada até $\mathrm{pH}=7$, seguida de secagem (105 $\left.{ }^{\circ} \mathrm{C}\right)$ por $24 \mathrm{~h}$.

Preparou-se solução de cloreto de zinco $\left(\mathrm{ZnCl}_{2}\right)$ que foi adicionada em béquer junto à biomassa já lixiviada e seca, na proporção 2:1 m/m (solução/biomassa) por $4 \mathrm{~h}$ sob agitação em banho Dubnoff-157 à temperatura de $30^{\circ} \mathrm{C}$. Em seguida, a matéria-prima foi levada para secagem à $105^{\circ} \mathrm{C}(24 \mathrm{~h})$.

A preparação do carvão se deu com a inserção da biomassa (com $\mathrm{ZnCl}_{2}$ ) em forno mufla à temperatura inicial de $200{ }^{\circ} \mathrm{C}$ por $1 \mathrm{~h}$. Em seguida, aumentou-se a temperatura para $300^{\circ} \mathrm{C}$ permanecendo por mais $1 \mathrm{~h}$. Repetiu-se o procedimento para a temperatura de $400{ }^{\circ} \mathrm{C}$ até a temperatura de $500{ }^{\circ} \mathrm{C}$, onde permaneceu por $2 \mathrm{~h}$. O processo de ativação química e carbonização foi realizado sob taxa de aquecimento de $17{ }^{\circ} \mathrm{C} \cdot \mathrm{min}^{-1}$ e vaso cerâmico.

Aguardou-se o arrefecimento do material e vaso cerâmico até a temperatura próxima à $50{ }^{\circ} \mathrm{C}$, onde foi realizada sua lavagem com solução de ácido clorídrico $(\mathrm{HCl})$ a $1 \mathrm{~N}$ para realizar a retirada de $\mathrm{ZnCl}_{2}$ presente na superfície do carvão. $\mathrm{O}$ material foi levado à estufa e seco à $105^{\circ} \mathrm{C}$ por $24 \mathrm{~h}$, assim, obtendo o carvão ativado a partir do endocarpo de tucumã (CATC). 


\section{Caracterização}

O carvão ativado foi submetido a análises físico-químicas com a finalidade de se obter conhecimento e/ou verificar suas propriedades físicas e químicas que auxiliam no estudo de adsorção e possível aplicação do mesmo como potencial adsorvente.

\section{Umidade}

Pesou-se $1 \mathrm{~g}$ das amostras de carvão ativado (análise realizada em triplicata) em cápsulas de porcelana com tampa. O conjunto cápsula + carvão foi colocado em estufa com circulação de ar a $150{ }^{\circ} \mathrm{C}$ por $3 \mathrm{~h}$. Ao final deste processo, as cápsulas foram tampadas e armazenadas em dessecador até atingir a temperatura ambiente e serem pesadas. O conteúdo de umidade foi calculado a partir da Equação 1 e realizado conforme a norma ASTM D2867-17.

$$
U(\% \text { em peso })=\left(\frac{m_{1}-m_{3}}{m_{1}-m_{2}}\right) \times 100
$$

Onde:

$\mathrm{m}_{1}$ : massa da cápsula mais a tampa $[\mathrm{g}]$; $\mathrm{m}_{2}$ : massa da cápsula mais a tampa e amostra [g]; $\mathrm{m}_{3}$ : massa da cápsula mais a tampa e amostra seca [g].

\section{Cinzas}

Calcinou-se cadinhos (análise realizada em triplicata) em forno mufla a $650 \pm 25^{\circ} \mathrm{C}$ por $1 \mathrm{~h}$. Os recipientes foram colocados em dessecador para que fossem resfriados e em seguida pesados. Paralelamente, as amostras de carvão ativado foram secas em estufa a $150 \pm 5^{\circ} \mathrm{C}$ por $3 \mathrm{~h}$. Ao final de ambos os processos, foram pesados em balança analítica 0,1 g dos CAs nos cadinhos calcinados e estes conjuntos foram levados ao forno mufla à $650 \pm 25$ ㄷ $\mathrm{C}$ por $3 \mathrm{~h}$ sob presença de oxigênio. Após este período, o conjunto foi arrefecido em dessecador até serem pesados e conhecido o teor de cinzas da amostra (Equação 2).

$$
\operatorname{Cinzas}(\%)=\left(\frac{m_{a}-m_{c}}{m_{a}-m_{b}}\right) x 100
$$

Onde:

$\mathrm{m}_{\mathrm{a}}$ : massa do cadinho antes da calcinação [g]; $\mathrm{m}_{\mathrm{b}}$ : massa do cadinho mais a massa de carvão antes da calcinação [g]; $\mathrm{m}_{\mathrm{c}}$ : massa do cadinho mais a massa de carvão após a calcinação $[\mathrm{g}]$.

\section{Grupos funcionais}

A metodologia de Boehm (1994) foi utilizada para a determinação e quantificação dos grupos funcionais, como: grupos carboxílicos $(-\mathrm{COOH})$, fenólicos $(-\mathrm{OH})$ e lactonas $(-\mathrm{COOR})$; presentes na superfície do $\mathrm{CACT}$, na qual consiste em adicionar o material adsorvente em soluções de $\mathrm{NaOH}, \mathrm{HCl}, \mathrm{Na}_{2} \mathrm{CO}_{3}, \mathrm{NaHCO}_{3}$ e submetidos a titulação para verificar a presença e quantificar grupos ácidos e básicos nas amostras. 


\section{Determinação de grupos ácidos (GA)}

Foram adicionados 2,0 g de carvão ativado a $25 \mathrm{~mL}$ de solução padrão de hidróxido de sódio $(\mathrm{NaOH})$ 0,1 M; carbonato de sódio $\left(\mathrm{Na}_{2} \mathrm{CO}_{3}\right)$ 0,1 $\mathrm{N}$ e bicarbonato de sódio ( $\mathrm{NaHCO}$ ) 0,1 N. Estas misturas foram realizadas em erlenmeyers $(100 \mathrm{~mL})$ que foram vedados com papel filme plástico PVC e submetidos a agitação de $140 \mathrm{rpm}$ por $24 \mathrm{~h}$ a $30^{\circ} \mathrm{C}$. Após este tempo (equilíbrio) a mistura foi filtrada à vácuo e foi retirada uma alíquota de $10 \mathrm{~mL}$ de cada solução $\left(\mathrm{NaOH}, \mathrm{Na}_{2} \mathrm{CO}_{3}\right.$ e $\left.\mathrm{NaHCO}_{3}\right)$, em que se foi titulado $\mathrm{HCl} 0,1 \mathrm{~N}$ utilizando fenolftaleína como indicador. A quantificação dos grupos funcionais ácidos foi realizada a partir da Equação 3.

$$
\mathrm{m}_{\mathrm{Eq}_{-} \mathrm{g}}(\mathrm{GA})=\frac{\mathrm{V}_{\mathrm{TA}} \cdot \mathrm{N}_{\mathrm{HCl}} \cdot\left(\mathrm{V}_{\mathrm{am}}-\mathrm{V}_{\mathrm{HCl}}\right)}{\mathrm{V}_{\mathrm{al}}}
$$

Onde: $V_{\text {TA: }}$ volumes de $\mathrm{NaOH}, \mathrm{Na}_{2} \mathrm{CO}_{3}$ e $\mathrm{NaHCO}_{3}$ utilizados na adsorção (antes da filtração) [mL]; $\mathrm{N}_{\mathrm{HCl}}$ : concentração de $\mathrm{HCl}$ utilizada na titulação [Eq/L].

\section{Determinação de grupos básicos (GB)}

2,0 g de CACT foram adicionados a $25 \mathrm{~mL}$ de solução padrão $\mathrm{HCl}$ 0,1 $\mathrm{N}$ em erlenmeyer de $100 \mathrm{~mL}$. Em seguida, o recipiente foi vedado com plástico filme PVC e submetido a agitação de $140 \mathrm{rpm}$ por $24 \mathrm{~h}$ a $30^{\circ} \mathrm{C}$.

\section{Espectroscopia na região do Infravermelho}

A identificação de grupos funcionais orgânicos presentes na superfície do carvão ativado produzido a partir do endocarpo de tucumã foi realizada com auxílio da técnica de espectroscopia vibracional na região do infravermelho utilizando transformada de Fourier (FTIR). A técnica consiste na absorção da radiação através de vibrações de átomos e moléculas em uma faixa ou estiramento característico.

\section{Ponto de carga zero $\left(\mathrm{pH}_{\mathrm{PCZ}}\right)$}

Esta análise foi realizada utilizando o método de 11 pontos. Foram-se ajustados os pH de soluções, com $\mathrm{HCl}$ ou $\mathrm{NaOH}$, em 1, 2, 3, 4, 5, 6, 8, 9, 10, 11 e 12 e em cada frasco também foram adicionados $50 \mathrm{mg}$ de CACT junto a $50 \mathrm{~mL}$ de solução. Em seguida, estas suspensões foram submetidas à agitação, em banho Dubnoff-157, de $80 \mathrm{rpm}$ por $24 \mathrm{~h}$ a $30^{\circ} \mathrm{C}$.

As amostras foram filtradas em papel filtro e utilizou-se $\mathrm{pHmetro} \mathrm{para} \mathrm{a} \mathrm{determinação} \mathrm{do} \mathrm{pH}$ final. $\mathrm{O}$ valor de $\mathrm{pH}_{\mathrm{pcz}}$ foi conhecido através da construção do gráfico $\mathrm{pH}$ inicial versus $\mathrm{pH}$ final.

\section{RESULTADOS E DISCUSSÃO}

O conteúdo de umidade apresentado pelo CATC foi de 6,12 \%. Este resultado elevado pode ser explicado pela temperatura relativamente baixa de ativação $\left(500{ }^{\circ} \mathrm{C}\right)$ e ainda assim, permanece abaixo do limite divulgado pela American Water Works Association (AWWA) B604/2012 que é de $8 \%$. A análise do conteúdo de cinza total do CATC foi realizada em triplicata e valor igual a 7,79\%. 
Os resultados da análise de grupos funcionais de superfície do carvão ativado do caroço de tucumã, utilizando a metodologia de Boehm (1994), estão descritos na Tabela 1. Esta análise permitiu a determinação e quantificação desses grupos. Valores acima dos encontrados por Zubir et al. (2020): 0,65 e 0,217.

Tabela 1: Grupos funcionais de superfície presentes no carvão ativado do caroço de tucumã.

\begin{tabular}{l|l|l}
\hline Amostra & Total de grupos ácidos $\left(\mathbf{m E q} \cdot \mathbf{g}^{-1}\right)$ & Total de grupos básicos $\left(\mathbf{m E q}^{\left.-\mathbf{g}^{-1}\right)}\right.$ \\
\hline CATC & 2,20 & 1,59 \\
\hline
\end{tabular}

A análise dos espectros foi realizada à temperatura ambiente na região de 4000 a $500 \mathrm{~cm}^{-1}$, ilustrados na Figura 1, permitindo a identificação dos grupos funcionais de superfície do CATC mostrados na Tabela 2.

As bandas 2922 e $2852 \mathrm{~cm}^{-1}$ indicam alongamento de grupo $\mathrm{CH}\left(\mathrm{CH}_{2}\right)$. Enquanto em $1744 \mathrm{~cm}^{-1}$ aponta alongamento $\mathrm{C}=\mathrm{O}$ e $\mathrm{C}=\mathrm{C}$. Em 1744 e $1562 \mathrm{~cm}^{-1}$ indicam a presença de grupos funcionais $\mathrm{C}=\mathrm{O}, \mathrm{C}=\mathrm{C}$, estiramento $\mathrm{O}-\mathrm{H}$ e como possíveis compostos ácidos carboxílicos, cetonas e outros. Alongamento C-O e estiramento O-H são indicados pelas bandas 1149 e $1021 \mathrm{~cm}^{-1}$. Compostos aromáticos são apresentados pelo estiramento $\mathrm{C}-\mathrm{H}$ e $\mathrm{N}-\mathrm{H}$ nas bandas 843 e $716 \mathrm{~cm}^{-1}$.

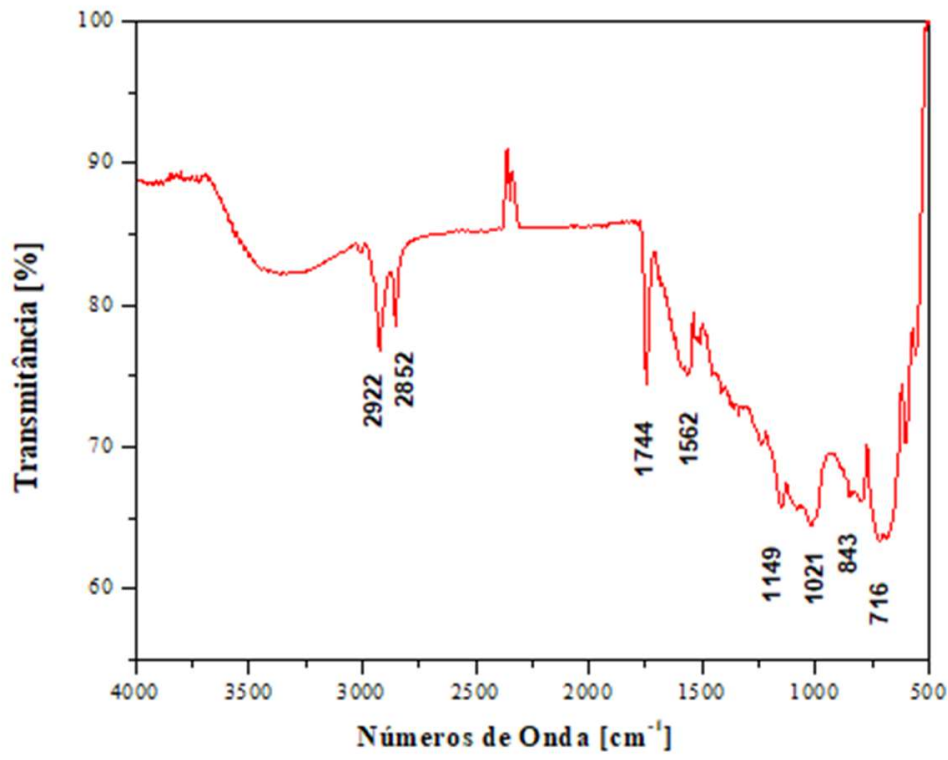

Figura 1: Espectros da análise no infravermelho.

Tabela 2: Bandas da análise FTIR e grupos funcionais.

\begin{tabular}{|l|l|l|l|}
\hline $\begin{array}{l}\text { Números de onda } \\
\left(\mathbf{c m}^{-1}\right)\end{array}$ & $\begin{array}{l}\text { Picos }\left(\mathbf{c m}^{-}\right. \\
\mathbf{1})\end{array}$ & Grupos funcionais & Compostos \\
\hline $\begin{array}{l}2843-2863 ; \quad 2916- \\
2936\end{array}$ & $\begin{array}{l}2922, \\
2852\end{array}$ & Alongamento CH & Alcanos \\
\hline 1744 & 1744 & Alongamento C=O & Éster \\
\hline $1500-1845$ & $\begin{array}{l}1744, \\
1562\end{array}$ & $\begin{array}{l}\text { C=O, C=C, estiramento } \\
\text { O-H }\end{array}$ & $\begin{array}{l}\text { Ácidos carboxílicos, aldeídos, anel aromático, cetonas, ácidos } \\
\text { carboxílicos }\end{array}$ \\
\hline $950-1300$ & $\begin{array}{l}1149, \\
1021\end{array}$ & $\begin{array}{l}\text { Alongamento C-O } \\
\text { Estiramento O-H }\end{array}$ & Álcoois primário, secundário e terciário, éteres, ésteres \\
\hline $650-1000$ & 843,716 & $\begin{array}{l}\text { Estiramento } \\
\text { Estiramento N-H }\end{array}$ & Grupos aromáticos \\
\hline
\end{tabular}

Fonte: Adaptado de Kumar et al. (2015).

$\mathrm{O}$ valor do $\mathrm{pH}$ no ponto de carga zero $\left(\mathrm{pH}_{\mathrm{PCZ}}\right)$ foi determinado a partir da Figura 2. O $\mathrm{pH}$ PCZ do CATC encontrado foi de 5,54, o que confirma a análise dos grupos funcionais de superfície do material adsorvente. Zubir et al. (2020) encontraram o valor de $\mathrm{pH}_{\mathrm{PCZ}}$ de 6,25 para o carvão produzido a partir de galhos de 
Pterocarpus indicus e ativado também como $\mathrm{ZnCl}_{2}$ e $\mathrm{Mu}^{\prime}$ azu et al. (2020) determinaram que lodo de esgoto ativado com $\mathrm{ZnCl}_{2}$ apresentou $\mathrm{pH}_{\mathrm{PCZ}}$ de 6,54.

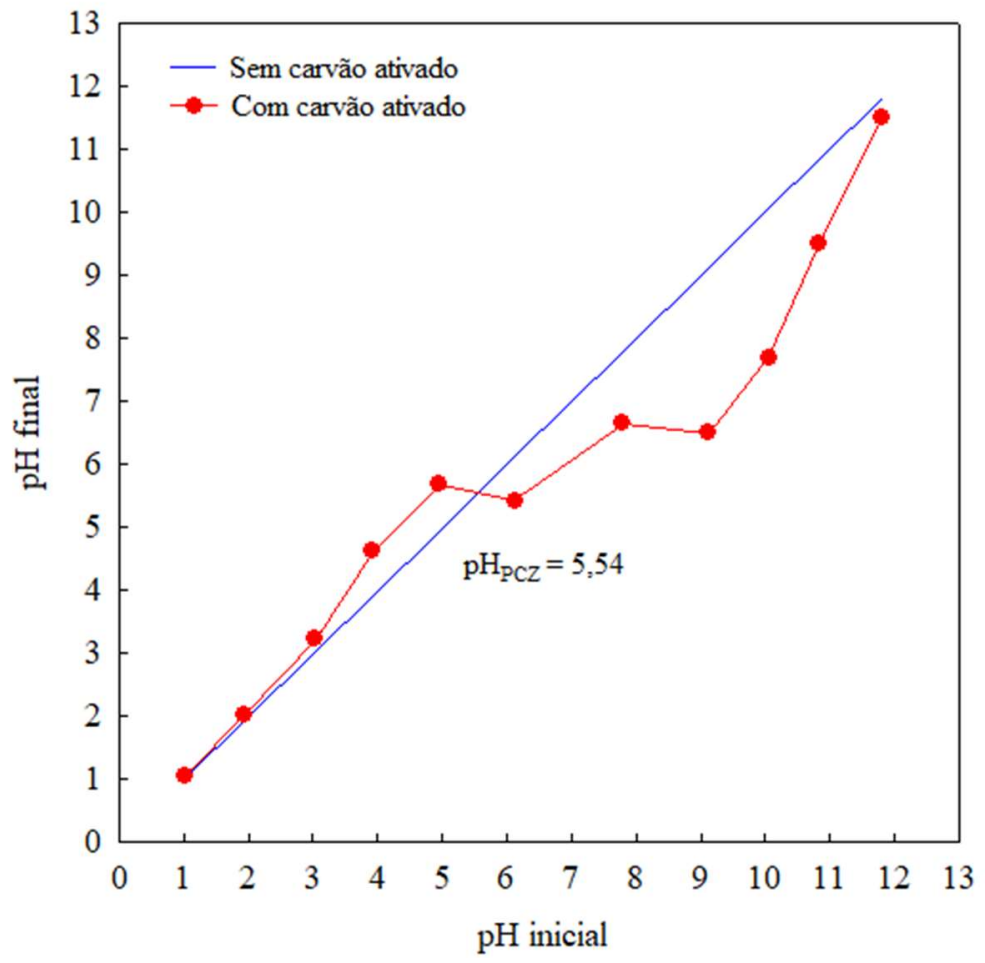

Figura 2: Determinação do pH no ponto de carga zero do CATC.

\section{CONCLUSÕES}

O carvão ativado produzido a partir do caroço de tucumã (CATC), utilizando $\mathrm{ZnCl}_{2}$ como ativante químico, apresentou grupos funcionais de superfície elevados e predominantemente ácidos que resultou em pH no ponto de carga zero igual a 5,54 e presença de diversos compostos como ácidos carboxílicos, cetonas e outros. O conteúdo de cinzas se mostrou elevado, mas dentro do aceitável, bem como o valor de umidade. O carvão ativado produzido a partir do endocarpo de tucumã apresentou características interessantes para aplicação na remoção de poluentes em efluentes líquidos, principalmente pela baixa temperatura de ativação/carbonização que torna o processo mais viável.

\section{REFERÊNCIAS}

BOEHM, L. E.. The Validity Effect: a search for mediating variables. Personality and Social Psychology Bulletin, v.20, n.3, p.285-293, 1994. DOI: http://dx.doi.org/10.1177/0146167294203006

KUMAR, A.; JENA, H. M.. High surface area microporous activated carbons prepared from Fox nut (Euryale ferox) shell by zinc chloride activation. Applied Surface Science, v.356, p.753-761, 2015. DOI:

http://dx.doi.org/10.1016/j.apsusc.2015.08.074

MU'AZU, N. D.; ZUBAIR, M.; JARRAH, N.; ALAGHA, O.; AL-
HARTHI, M. A.; ESSA, M. H.. Sewage Sludge ZnCl2-Activated Carbon Intercalated MgFe-LDH Nanocomposites: insight of the sorption mechanism of improved removal of phenol from water. International Journal of Molecular Sciences, v.21, n.5, p.1563, 2020. DOI: http://dx.doi.org/10.3390/ijms21051563

ZUBIR, M. H. M.; ZAINI, M. A. A.. Twigs-Derived Activated Carbons via $\mathrm{H} 3 \mathrm{PO} 4 / \mathrm{ZnCl} 2$ Composite Activation for Methylene Blue and Congo Red Dyes Removal. Scientific Reports, v.10, n.1, p.14050, 2020. DOI: https://doi.org/10.1038/s41598-020-71034-6

A CBPC - Companhia Brasileira de Produção Científica (CNPJ: 11.221.422/0001-03) detém os direitos materiais desta publicação. Os direitos referem-se à publicação do trabalho em qualquer parte do mundo, incluindo os direitos às renovações, expansões e disseminações da contribuição, bem como outros direitos subsidiários. Todos os trabalhos publicados eletronicamente poderão posteriormente ser publicados em coletâneas impressas sob coordenação da Sustenere Publishing, da Companhia Brasileira de Produção Científica e seus parceiros autorizados. Os (as) autores (as) preservam os direitos autorais, mas não têm permissão para a publicação da contribuição em outro meio, impresso ou digital, em português ou em tradução. 